\title{
Total body irradiation in a case of thalassemia major with source to axis distance based planning: A case report
}

\author{
Dr. Mayuresh D. Virkar', Mr. Rajkumar Chauhan ${ }^{2}$, DR. Pranav Chadha ${ }^{3}$, Dr. Kaustav Talapatra ${ }^{4}$, \\ Dr. Reuben Jake Rodrigues ${ }^{5}$, Dr. Amrita Shrivastava ${ }^{6}$, Dr. Santanu Sen ${ }^{7}$, Dr. Sameer Tulpule ${ }^{8}$
}

\begin{abstract}
${ }^{1}$ Clinical Associates, Radiation Oncology Dept., Kokilaben Dhirubhai Ambani Hospital, Mumbai, India ${ }^{2}$ RSO, Medical Physicist, Radiation Oncology Dept., Kokilaben Dhirubhai Ambani Hospital, Mumbai, India 3Head of the Unit, Radiation Oncology Dept., Kokilaben Dhirubhai Ambani Hospital, Mumbai, India ${ }^{4}$ Head of the Department, Radiation Oncology Dept., Kokilaben Dhirubhai Ambani Hospital, Mumbai, India 5Medical Officer, Radiation Oncology Dept., Kokilaben Dhirubhai Ambani Hospital, Mumbai, India ${ }^{6}$ DMRT, Radiation Oncology Dept., Kokilaben Dhirubhai Ambani Hospital, Mumbai, India ${ }^{7,8}$ Consultant, Haematology Dept., Kokilaben Dhirubhai Ambani Hospital, Mumbai, India
\end{abstract}

\begin{abstract}
Background: The use of total body radiation (TBI) before hematopoietic stem cell transplantation (HSCT) would increase the engraftment without transplant-related morbidity or mortality among Thalassemia major (TM) cases. Case presentation: A 2-year-old female child, diagnosed with TM was scheduled for haploidentical allogenic transplant-based protocol, and after that, based on protocol she was scheduled to undergo a single session of $\mathrm{TBI}$ as a conditioning regimen before haploidentical allogenic hematopoietic stem cell tranplant. A total dose of 4 Gy was administered.. The incidence of graft failure was reduced as TBI was used before allogeneic stem cell transplantation. TBI provided a uniform dose of radiation to the entire body, penetrating areas such as the central nervous system (CNS) and testes. Conclusion: Total Body Irradiation with the SAD technique is the most effective way of treatment. As it is comfortable for the patient to undergo, easily reproducible, and it helps to achieve a uniform dose distribution.
\end{abstract}

KEYWORDS: Hematopoietic Stem Cell Transplantation; Graft-versus-Host Disease; Thalassemia Minor; Radiotherapy; Myeloablative conditioning; Haemoglobinopathies

\section{INTRODUCTION}

$\mathrm{T}$ Thalassemia major (TM), inherited anaemia that is associated with reduced or absent beta-globin synthesis, results in an imbalanced accumulation of beta-globin chains and ineffective erythropoiesis with haemolysis [1].

Thus, the curative mode of therapy remains the replacement of the defective erythropoiesis by allogeneic hematopoietic stem cell transplantation (HSCT). Due to the high chances of graft failure, various novel conditioning regimens were used to limit the toxicity from myeloablative conditioning regimens and graft-versus-host disease (GVHD) $[2,3]$.

Conditioning regimens can be in the form of chemotherapy and Total Body radiotherapy.

Correspondence: DR. Pranav Chadha, HOU, Radiation Oncology Dept., Kokilaben Dhirubhai Ambani Hospital, Mumbai, Maharashtra, India.Email: radpranav@gmail.com

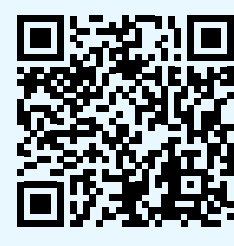


The use of total body radiation (TBI) before hematopoietic stem cell transplant would increase the engraftment without transplantrelated morbidity or mortality.

\section{Case report}

A 2-year-old female child, diagnosed with TM, was admitted and planned for haploidentical allogenic transplant (Rabbit ATG/Thiotepa/ FLU/Cy/TBI/PTCy) based protocol from August 22, 2019, to August 28, 2019. She was scheduled to undergo a single session of TBI on August 29, 2019, as a component of the protocol.

The CT planning procedure was executed on August 22, 2019 employing short sedationanesthetizing the child followed by immobilization in vac-loc. A whole-body CT scan $(3 \mathrm{~mm}$ slice cuts) from the vertex to $2 \mathrm{~cm}$ beyond the toes was acquired for the radiation planning and dosimetry.

A total dose of 4 Gy was administered as per the protocol.

The following was the modus operandi:

The patient was treated under anaesthesia. In this particular case, a source-axis distance (SAD) field plan was utilized to treat the patient employing Varian Clinac iX (Trilogy). The advantages of this approach include the ability to treat patients. At the same time, they are supine, which makes the treatment more comfortable, an isocentric approach that does not require further setup corrections as in source-surface distance (SSD) technique, and greater reproducibility. Wholebody CT scans, with the patient in the treatment position, were used for treatment planning, and multileaf collimator fields were used.

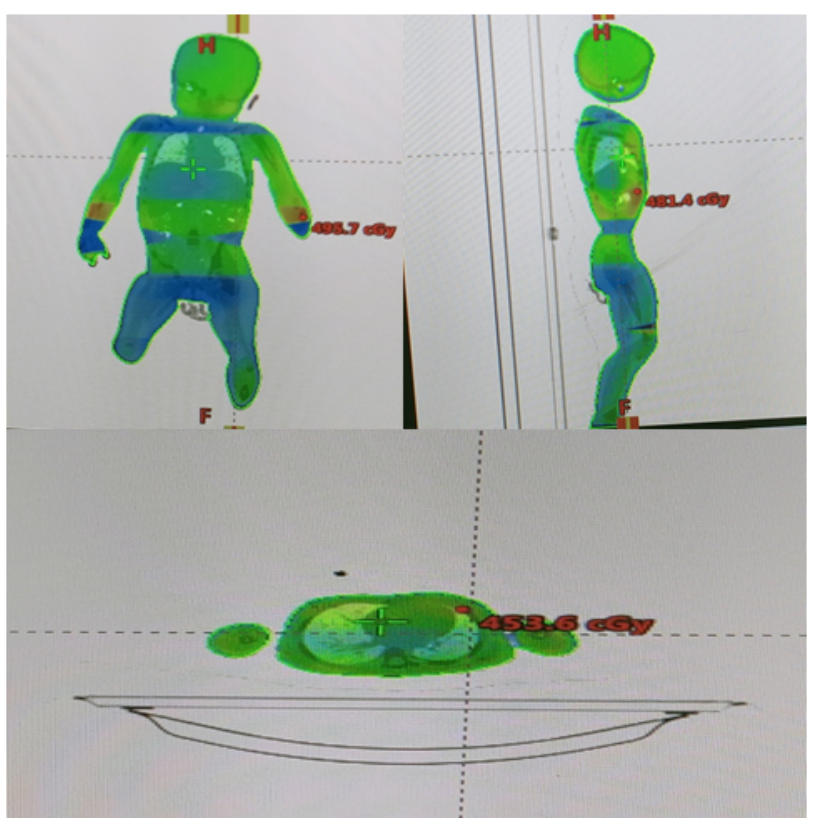

Fig 1. Dose Colour Wash
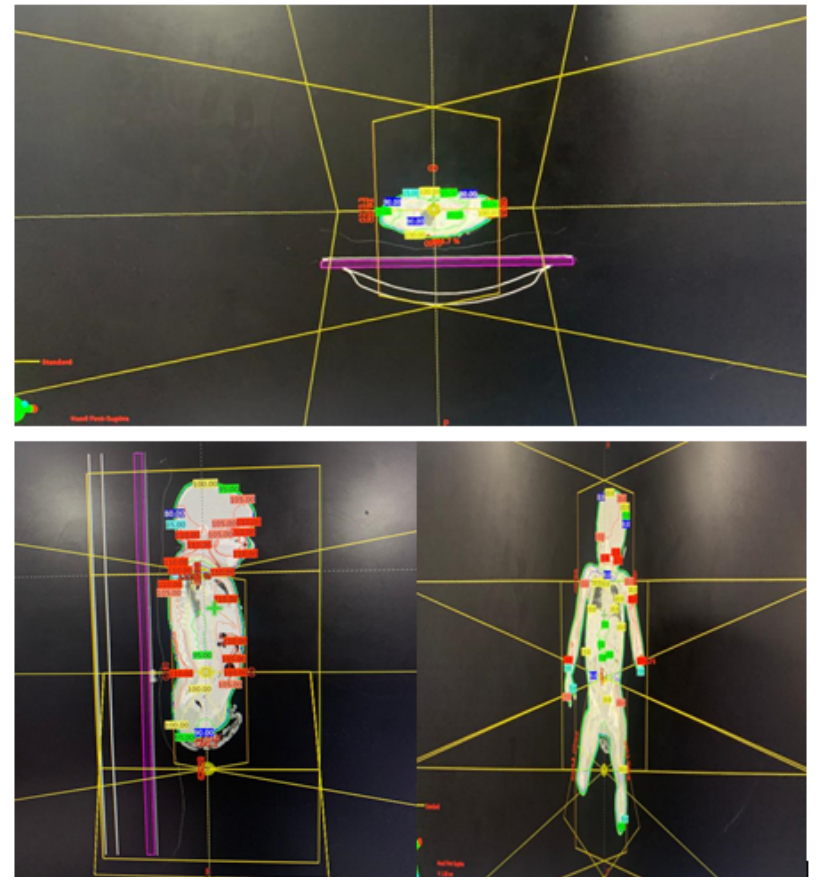

Fig 2. Beam's eye view

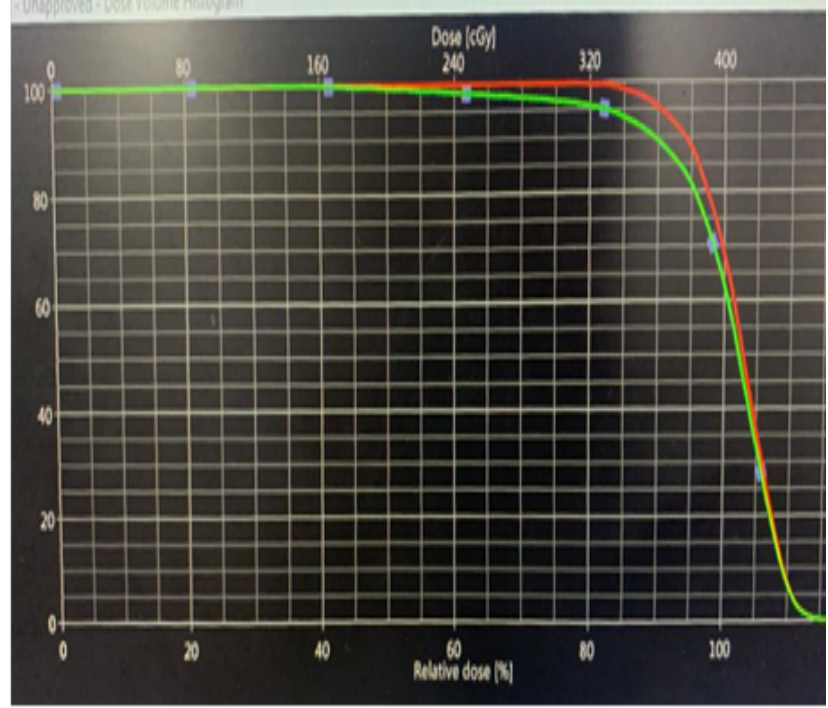

Fig 3. DVH

The brain, lungs, and kidneys were not contoured intentionally because a single dose does not seem to be associated with significant acute side effects and is well tolerated. A Field in Field plan was created for the treatment; the brain was treated by 2 bilateral fields $\left(90^{\circ}\right.$ $\left.270^{\circ}\right)$. A four-field planning technique was used to treat the rest of the body, taking care of the overlapping parts of the jaws, and a few fields in fields were used to compensate for the cold spots. Dose distribution in all planes is shown in figures. (Figure no. 1,2 \& 3).

\section{Discussion}

Haemoglobin disorders constitute the most common class of monogenic disorders in the world, with 330,000 affected infants born 
annually, $17 \%$ of which suffer from thalassemias [1]. The combined use of both erythrocyte transfusion and iron-chelating therapy has resulted in a remarkable improvement in the life expectancy of patients with TM $[2,3]$. However, complications related to iron overload cannot be completely managed through chelating therapy and chronic transfusion regimens, as they are difficult to maintain throughout a lifetime. Thus the safe and only curative therapy remains the replacement of the defective erythropoiesis by allogeneic hematopoietic stem cell transplantation (HSCT) [4,5].

Historically, allogeneic hematopoietic stem cell transplantation for severe hemoglobinopathies required myeloablative conditioning to enable engraftment. Accordingly, the broader application of allogeneic blood or marrow transplantation in severe Beta-hemoglobinopathies depends on novel strategies that address the issues of donor availability and limit the toxicity from myeloablative conditioning regimens and graftversus-host disease (GVHD).

However, in order to prevent graft failure, various strategies were used, such as administration of cyclophosphamide, which results in a low incidence of GVHD. In order to increase the engraftment, Total Body Irradiation should be used before allogeneic stem cell transplantation, followed by conditioning chemotherapy as this protocol has a reduced incidence of graft failure. According to a study done at Johns Hopkins Hospital, increasing the dose of total body irradiation from 200cGy to 400cGy allowed stable engraftment [6]. (4Gy) was used in our patient, who is a diagnosed case of Beta-thalassemia major. However, in this patient SAD technique was used for the delivery of treatment.

Total body irradiation (TBI) with megavoltage photon beams is used as one component before autologous stem cell transplant for treating several diseases, including multiple myeloma, leukaemia's, lymphomas etc. $[7,8]$.

TBI is uniform dose of radiation to the entire body including cen $\neg$ tral nervous system (CNS) and testes, where traditional chemotherapy is inef $\neg$ fective $[9,10]$. Additionally, TBI allows one to tailor the therapy with the use of shields or by boosting the dose to specific regions as necessary. The purpose of TBI is to eliminate residual cancer cells and to provide space for stem cell engraft $\neg$ ment through bone marrow depletion, and to prevent rejection of donor stem cells through immunosuppression $[11,12]$. Numerous techniques have been used to deliver TBI, the commonly used technique being AP (anteroposterior)-PA (posteroanterior) because body separation varies little less than lateral body separation; Typically AP separation is between 20 and $25 \mathrm{~cm}$ for adults, while lateral body separation has a maximum value up to about $50 \mathrm{~cm}$. AAPM Report No.17 recommends an AP-PA TBI technique. Generally combining AP-PA and Bilateral fields is not practiced because of the difficulties of field setup. Thus better dose uniformity along the longitudinal body axis is achieved by AP-PA technique, but the patient positioning may cause problems. One of the methods of delivering TBI is with the patient standing or lying down at an extended source-to-skin distance (SSD). The entire patient is usually treated with a single radiation beam, with a large (approximately $4 \mathrm{~m}$ ) SSD and the largest practical field size. The large SSD reduces the dose rate to about $10 \%$ of that typically used in RT. A typical field arrangement is to have the patient standing, to rotate the collimator by 45 degrees to use the diagonal dimension of the field, to achieve a uniform dose rate from head to foot lead strips are used as compensating filters, to use a Plexiglas barrier close to the patient to defeat the skin-sparing effect of highenergy photons, and to block critical organs in order to reduce the dose to these tissues. Anterior and posterior fields are used with the dose prescribed to mid-plane [13]. The patients typically stand on a stand designed for TBI or lie on a specially designed table at floor level. Most centres use photon energies between 6 and $10 \mathrm{MV}$. A beam spoiler of low atomic number material is placed close to the patient because of the skin-sparing effects of photons. In our institute, we use $6 \mathrm{MV}$ photons to avoid underdosing the skin and superficial bones such as the iliac crest and sternum. AAPM's TG-51and TRS 398 calibration protocol provides guidelines for dosimetry of high-energy photon beams. The efficacy of dynamic arc-based techniques and helical tomotherapy is demonstrated for decreasing TBI treatment time and in $\neg$ creasing homogeneity of delivered radiantion; however, the use of this technique is not widespread.

Linear accelerators were commissioned using the source-to-surface dis 7 tance $(\mathrm{SSD}=428 \mathrm{~cm})$. The absolute dose was calibrated at $100 \mathrm{~cm}$ SSD (surface to axis dis 7 tance) using a 10-x-10$\mathrm{cm}$ field size ac 7 cording to the AAPM TG-51 protocol, but TBI treatments are delivered using a larger field $(40-\mathrm{x}-40-\mathrm{cm})$ and extended SSD. Thus the dosimetry tasks for TBI commissioning include: 1) measuring the output factor at the central point of treatment distance; 2) generating the table of maximum tissue ratio (TMR) at the central point of treatment distance; This was performed using a PTW (PPC40) ion chamber (PTW, Freiburg, Germany), a Fluke electrom 7 eter (Fluke Biomedical, Everett, Wash 7 ington), and multiple $30-x-30-\mathrm{cm}$ PVC phantoms. To independently verify dosimetric 
accuracy, in-vivo mea $\neg$ surements with nanoDot OSLD do 7 simeters were performed with PVC phantoms after commissioning, and c) measuring two-dose profiles - one longitudinal and one transverse to the body midline, an ionization chamber with a suitable small build-up cap simulating a tissue depth of $10 \mathrm{~cm}$ is scanned through the beam which is free in the air. A single source of radiation is used; the patient is rotated 180 degrees along the beam central axis. For each field, the coronal midline of the patient is aligned with the crosswire and umbilicus of the patient. Better dose uniformity is provided along the anteri $\neg$ or-posterior/posterior-anterior (AP/ PA) direction. TBI stands were used to immobilize the patient-facing chest to beam central axis. Different setups were used for the treatment of TBI patients. Certain types of diseases like leukemias that can circulate in the blood volume of the skin are treated with TBI where full dose of radiation is preferable on skin and it is achieved with the help of beam spoiler, thus allowing energy to de $\neg$ posit near the surface of the skin.

Lung shielding using lead or alloy attenuators, which reduce radiation to the Lungs was used. Shielding is very precise if shields are directly fixed to the skin. The position of shields has to be verified under treatment conditions prior to each field. Bilateral TBI alone causes a low dose to mediastinum, ribs, and arms.

Thus there has been increase in number of centres have begun to use CT based 3-D planning, intensity-modulated radiation therapy (IMRT) to improve dose uniformity and to reduce dose to critical organs.

\section{Conclusion}

Total Body Irradiation with the SAD technique is the most effective way of treatment. As it is comfortable for the patient to undergo, easily reproducible, and it helps to achieve a uniform dose distribution. intervention lung patency will be maintained.

Conflict of interest: No conflict of interest. Informed Consent: A written informed consent to publish this case was obtained from the patient family.

\section{Funding: Nil}

\section{REFERENCES}

1. Modell B, Darlison M. Global epidemiology of haemoglobin disorders and derived service indicators. Bull World Health Organ. 2008;86(6):480-7

2. Neufeld EJ. Update on iron chelators in thalassemia. Hematology Am Soc Hematol Educ Program. 2010;451-5

3. Trachtenberg F, Vichinsky E, Haines D. Iron chelation adherence to deferoxamine and deferasirox in thalassemia. Am J Hematol. 2011; 86(5):433-6

4. Angelucci E, Baronciani D. Allogeneic stem cell transplantation for thalassemia major. Haematologica. 2008; 93(12):1780-4

5. Javier-Meade,Keneth Cooke Christopher Gamper. Effect of increased dose of total body irradiation on graft failure associated with HLA haploindentical. Lancet. 2019; S2352-3026 (30031-36)

6. Lucarelli G, Gaziev J. Advances in the allogeneic transplantation for thalassemia. Blood Rev. 2008;22(2):53-63

7. Sobecks RM, Dean R, Rybicki LA. 400 cGy TBI with fludarabine for reduced-intensity conditioning allogeneic hematopoietic stem cell transplantation. Bone Marrow Transplant. 2008; 42: 715-22

8. Levitt, SH, Purdy JA, Perez CA, eds. Technical Basis of Radiation Therapy: Practical Clinical Appli $\neg$ cations. Berlin, Germany: Springer. 2006;

9. Zheng Y, Dou Y, Duan L. Using chemo-drugs or irradiation to break immune tolerance and facilitate immunotherapy in solid cancer. Cellular Immunolᄀogy. 2015; 294(1):54-9

10. Halperin E, Constine L, Tarbell N, Kun L. Pediᄀatric Radiation Oncology. Philadelphia: Lippincott Williams \& Wilkins4th Edition; 2005.

11. Halperin E, Wazer D, Perez C, Brader L. Perez and Brady's Principles and Practice of Radiation Oncology. Philadelphia: Lippincott Wil liams \& Wilkins 6th Edition; 2013

12. Khan F. The Physics of Radiation Therapy. Philadelphia: Lippincott Williams \& Wilkins 3rd Edition; 2003.

13. Seung SK, Larson DA, Galvin JM, Mehta MP, Potters L, Schultz CJ et al. American College of Radiology (ACR) and American Society for Radiation Oncology (ASTRO) Practice Guideline for the Performance of Stereotactic Radiosurgery (SRS). Am J Clin Oncol. 2013;36(3):310-5 